\title{
Application of Raman Microscopy for Dairy Products Analysis
}

\author{
Aleksandar Nedeljković
}

\begin{abstract}
Raman microspectroscopy is applied for analysis of milk and dairy products.

The quality of spectra depends on type of the sample as well as on monitoring parameters. In the case of diluted samples problem can occure due to fluorescence of water. The choice of objective, duration of acquisition and temperature are essential for obtaining a good quality spectrum.
\end{abstract}

Raman spectroscopy, vibrational spectroscopic technique based on inelastic light scattering, provides qualitative and quantitative information about numerous types of samples and has therefore been applied in various research fields. Application of Raman spectroscopy in food science includes industrially oriented process and quality control, compositional analysis as well as more in-depth research utilization (examination of structure and structural changes of food components). Compared to another vibrational spectroscopic technique - IR absorption spectroscopy - Raman spectroscopy is more suitable for studying complex food systems, primarily due to the weak Raman scattering properties of water (Li-Chan 1996).

How to cite this book chapter:

Nedeljković, A. 2019. Application of Raman Microscopy for Dairy Products Analysis.

In: Vucelić Radović, B., Lazić, D. and Nikšić, M. (eds.) Application of Molecular

Methods and Raman Microscopy/Spectroscopy in Agricultural Sciences and Food

Technology, Pp. 171-175. London: Ubiquity Press. DOI: https://doi.org/10.5334/

bbj.m. License: CC-BY 4.0 
However, several questions arise during the course of Raman analysis. Therefore, in order to employ the full potential of this powerful technique and to acquire satisfactory spectra it is important to appropriately setup the Raman system by tuning several parameters (excitation wavelength i.e. laser, acquisition time, spectral range) and in addition to consider specific issues coming from the particular analyzed sample (Koca et al. 2010).

Raman spectroscopy is generally well-known for lack of sample preparation. As an illustration, for solid dairy products (cheeses, butter etc.) it is sufficient just to place the sample on the microscope slide. At the same time analysis of liquid dairy products (milk, yogurt etc.) requires employment of appropriate sample holder. Even though most of the Raman systems are equipped with specialized accessories for the analysis of liquid samples, where the cuvette is utilized, one can simply use custom-made sample container placed on microscope slide. For this purpose plastic cylinders are usually the best choice. Furthermore, usage of glass cuvette for analysis of milk has not shown best results because of the high turbidity of milk.

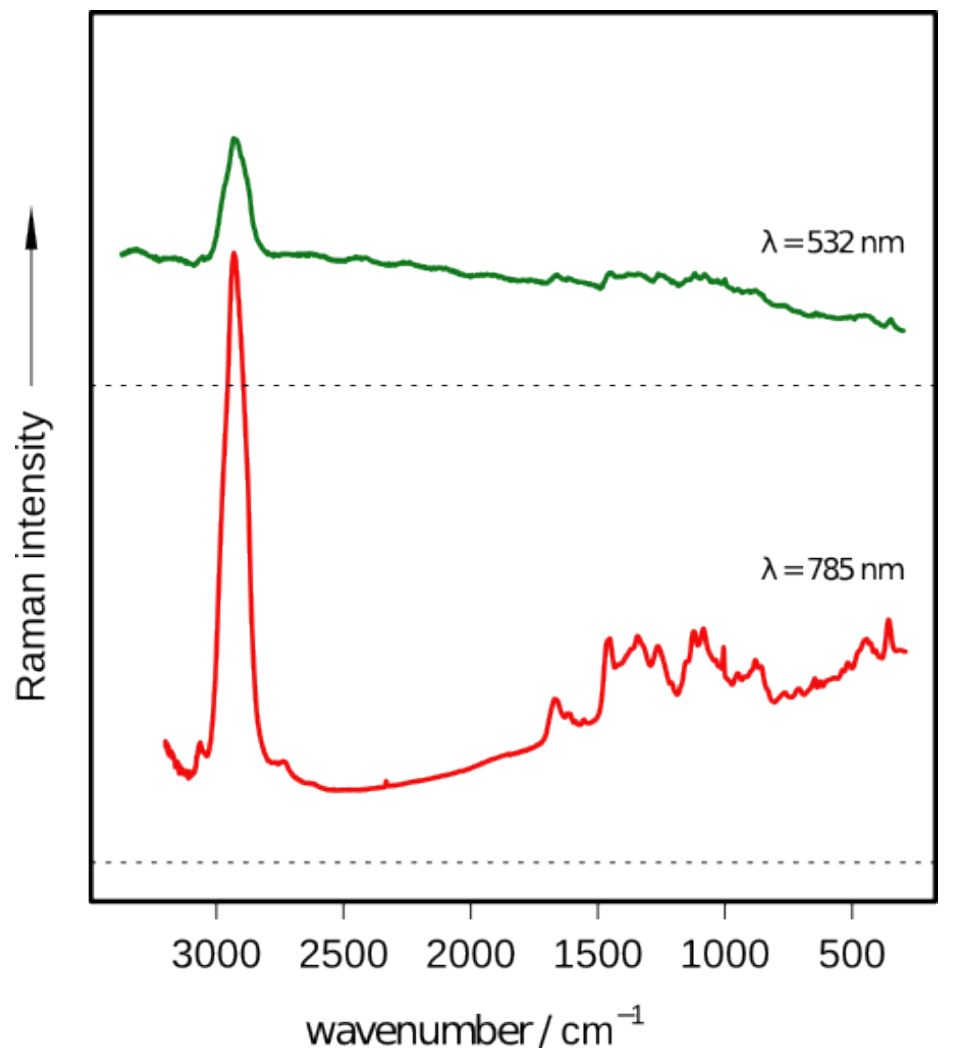

Figure 1: Fluorescence in analysis of milk samples. 
Major drawback of Raman spectroscopy is the occurrence of fluorescence, and in the case of milk and dairy products it occurs with $532 \mathrm{~nm}$ lasers. According to Nedeljković et al. (2016) the spectral region $1800-800 \mathrm{~cm}^{-1}$ is important for milk fat analysis, which is almost completely overlapped by fluorescence by laser at $532 \mathrm{~nm}$ (Figure 1). This drawback can be overcome by using lasers of a higher wavelength (785 nm, $1064 \mathrm{~nm}$ ) (Figure 1.), at the expense of lower peak intensities. However, with the selection of slightly longer acquisition time, highquality spectra can easily be acquired. Similarly, longer acquisition time (longer than $45 \mathrm{~s}$ ) is also required for analysis of milk (Figure 2.). As can be seen, the short acquisition time provides only strong intensity of the $\mathrm{CH}_{2}$ and $\mathrm{CH}_{3}$ vibrations (around $2900 \mathrm{~cm}^{-1}$ ), while increasing of the acquisition time enables clear visibility of peaks from milk sugars and proteins (Júnior et al. 2016) or from milk fat (Nedeljković et al. 2016). In contrast, with various "concentrated" milk systems (milk powders, cheese, kajmak etc.) where the water content is lower compared to milk, acquired spectra intensities are higher; hence acquisition time of $10-20 \mathrm{~s}$ is sufficient for satisfactory spectra.

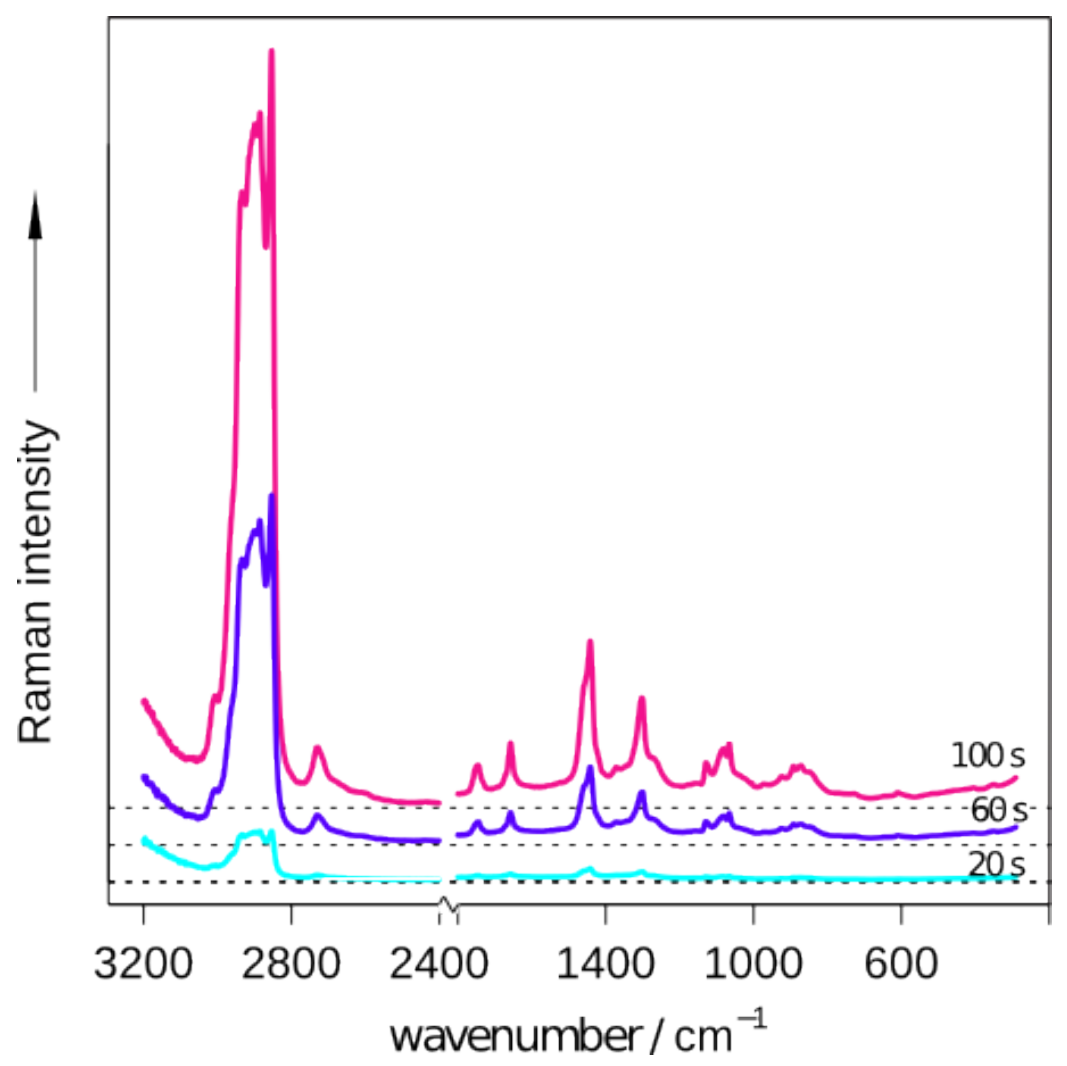

Figure 2: Influence of acquisition time on quality of milk spectra. 
Another important feature in the Raman analysis of dairy products is the selection of objective which is used to focus the laser light to the sample. Generally if the specific task of the analysis is to examine the bulk of dairy product, and not to address the specific micro-structural issue, it is better to use the objective of lower magnification $(\mathrm{x} 10, \mathrm{x} 5)$. The microscope can be used to get data successfully, but using small spot sizes can be very difficult for systems that can phase separate or contain domains of well-defined shape and refractive index - such as a lipid spheres i.e. milk fat globules. This can be addressed by taking multiple sample spot measurements on one sample or even mapping one of the samples to prove that domains are not a serious issue.

Finally, sample temperature during the spectra acquisition is quite important for Raman measurements (Abbas et al. 2009, Baeten et al. 2005). For that reason, consideration should be given to the selection of appropriate temperature and the selected temperature should be used throughout the given analysis. This is especially important for high-fat dairy products (cream, butter, highfat cheeses) since the structural properties of the milk fat change considerably with the change of temperature and since the part of spectra in the "fingerprint" region corresponds to physical state of milk fat. Nevertheless, it was observed that holding the sample at room temperature for approximately $30-60 \mathrm{~min}$ prior to analysis would give adequate results. In the case of other temperatures, it is necessary to use temperature control system.

\section{References}

Abbas, O., Fernández Pierna, J. A., Codony, R., von Holst, C., \& Baeten, V. (2009). Assessment of the discrimination of animal fat by FT-Raman spectroscopy. Journal of Molecular Structure, 924-926, 294-300. DOI: http:// dx.doi.org/10.1016/j.molstruc.2009.01.027

Baeten, V., Fernández Pierna, J. A., Dardenne, P., Meurens, M., GarcíaGonzález, D. L., \& Aparicio-Ruiz, R. (2005). Detection of the Presence of Hazelnut Oil in Olive Oil by FT-Raman and FT-MIR Spectroscopy. Journal of Agricultural and Food Chemistry, 53(16), 6201-6206. DOI: https://doi. org/10.1021/jf050595n

El-Abassy, R., Donfack, P., \& Materny, A. (2009). Rapid Determination of Free Fatty Acid in Extra Virgin Olive Oil by Raman Spectroscopy and Multivariate Analysis. Journal of the American Oil Chemists' Society, 86(6), 507-511. DOI: https://doi.org/10.1007/s11746-009-1389-0

Júnior, P.H.R., de Sá Oliveira, K., de Almeida, C.E.R., De Oliveira, L.F.C., Stephani, R., da Silva Pinto, M., de Carvalho, A.F., Perrone, I.T. (2016). FTRaman and chemometric tools for rapid determination of quality parameters in milk powder: Classification of samples for the presence of lactose and fraud detection by addition of maltodextrin. Food Chemistry, 196, 584588. DOI: https://doi.org/10.1016/j.foodchem.2015.09.055 
Koca, N., Kocaoglu-Vurma, N. A., Harper, W. J., \& Rodriguez-Saona, L. E. (2010). Application of temperature-controlled attenuated total reflectance-mid-infrared (ATR-MIR) spectroscopy for rapid estimation of butter adulteration. Food Chemistry, 121(3), 778-782. DOI: http://dx.doi.org/10.1016/j.foodchem.2009.12.083

Li-Chan, E. C. Y. (1996). The applications of Raman spectroscopy in food science. Trends in Food Science \& Technology, 7(11), 361-370. DOI: http:// dx.doi.org/10.1016/S0924-2244(96)10037-6

Nedeljković, A., Rösch, P., Popp, J., Miočinović, J., Radovanović, M., Pudja, P. (2016). Raman Spectroscopy as a Rapid Tool for Quantitative Analysis of Butter Adulterated with Margarine. Food Analytical Methods, 9, 1315-1320. DOI: https://doi.org/10.1007/s12161-015-0317-1 
